\title{
Solar-like Oscillations
}

\author{
Timothy R. Bedding ${ }^{1}$ and Hans Kjeldsen ${ }^{2,3}$ \\ ${ }^{1}$ School of Physics A28, University of Sydney, NSW 2006, Australia \\ bedding@physics.usyd.edu.au \\ ${ }^{2}$ Teoretisk Astrofysik Center, Danmarks Grundforskningsfond, 8000 Aarhus C, Denmark \\ hans@phys.au.dk \\ ${ }^{3}$ Institut for Fysik og Astronomi, 8000 Aarhus C, Denmark \\ Received 2003 May 12, accepted 2003 May 19
}

\begin{abstract}
The five-minute oscillations in the Sun have provided a wealth of information about the solar interior. After many attempts, positive detections of similar oscillations in solar-type stars have now been made. This review discusses the properties of solar-like oscillations, the methods used to observe them, and the results on individual stars. We conclude that the study of solar-like oscillations from the ground and space has an exciting future.
\end{abstract}

Keyword: stars: oscillations

\section{Introduction}

Measuring stellar oscillations is a beautiful physics experiment. A star is a gaseous sphere and will oscillate in many different modes when suitably excited. The frequencies of these oscillations depend on the density, temperature, gas motion, and other properties of the stellar interior. The amplitudes of the oscillations are determined by the excitation and damping processes, which may involve turbulence from convection, opacity variations, and magnetic fields. Studying the frequencies and amplitudes of oscillations in different types of stars promises to lead to significant advances in our understanding of stellar structure and evolution.

The best targets are stars which oscillate in several modes simultaneously. The frequency of each mode depends on the mode structure (e.g., the number of nodes) and on spatial variations of the sound speed and buoyancy frequency within the star. The frequency spectrum therefore places strong constraints on the internal structure. This analysis, called asteroseismology, yields information about composition, age, mixing and internal rotation that cannot be obtained in any other way and is completely analogous to the seismological study of the interior of the Earth. For reviews, see Brown \& Gilliland (1994) and Gautschy \& Saio $(1995,1996)$, and the proceedings of recent conferences (Aerts et al. 2002; Thompson et al. 2003).

The best-studied example of an oscillating star is the Sun. It oscillates in many modes simultaneously, with periods in the range 3-15 minutes. The frequencies of the modes contain information about the sound speed and rotation deep within the Sun, with each mode sensing these quantities in subtly different ways (e.g., Gough \& Toomre 1991; Christensen-Dalsgaard 2002; ESA 2002). Very accurate measurements of the frequencies have allowed inversion to determine the internal structure and rotation of the Sun. Helioseismology has provided tests of theoretical models of solar evolution and led to fascinating new insights into the complex dynamics of solar internal rotation. Observations that do not resolve the solar disk restrict us to observing modes of the lowest degree, but these still contain a great deal of information. The frequency spectrum of the Sun observed in integrated light is shown in Figure 1.

The solar oscillations are excited by convection near the surface, and it is therefore to be expected that all stars having such a convection zone will show similar oscillations. We use the term solar-like oscillations to refer to oscillations excited stochastically by convection. This presumably includes all stars cool enough to have an outer convection zone, from roughly the cool edge of the classical instability strip out to the red giants. However, at least in stars of roughly solar type, the expected amplitudes are tiny, which places extreme demands on instrumental stability. In recent years, however, success has finally come. In this review we will describe the main results that have been achieved so far and make some comments about future prospects.

\section{Properties of Oscillations}

Solar-like oscillations are standing sound waves, known as p modes (since pressure is the restoring force). Each mode is characterised by three integers: the radial order $n$, the angular degree $l$, and the azimuthal degree $m$. Mode frequencies for low-degree p-mode oscillations are approximated reasonably well by the asymptotic relation

$$
v_{n, l}=\Delta v\left(n+\frac{1}{2} l+\epsilon\right)-l(l+1) D_{0} .
$$

Here, $\Delta v$ (the so-called large separation) reflects the average stellar density, $D_{0}$ is sensitive to the sound speed near the core, and $\epsilon$ is sensitive to the surface layers. It is conventional to define $\delta v_{02}$, the so-called small separation, as the frequency spacing between adjacent modes with $l=0$ and $l=2$. These separations are shown in Figure 2, 


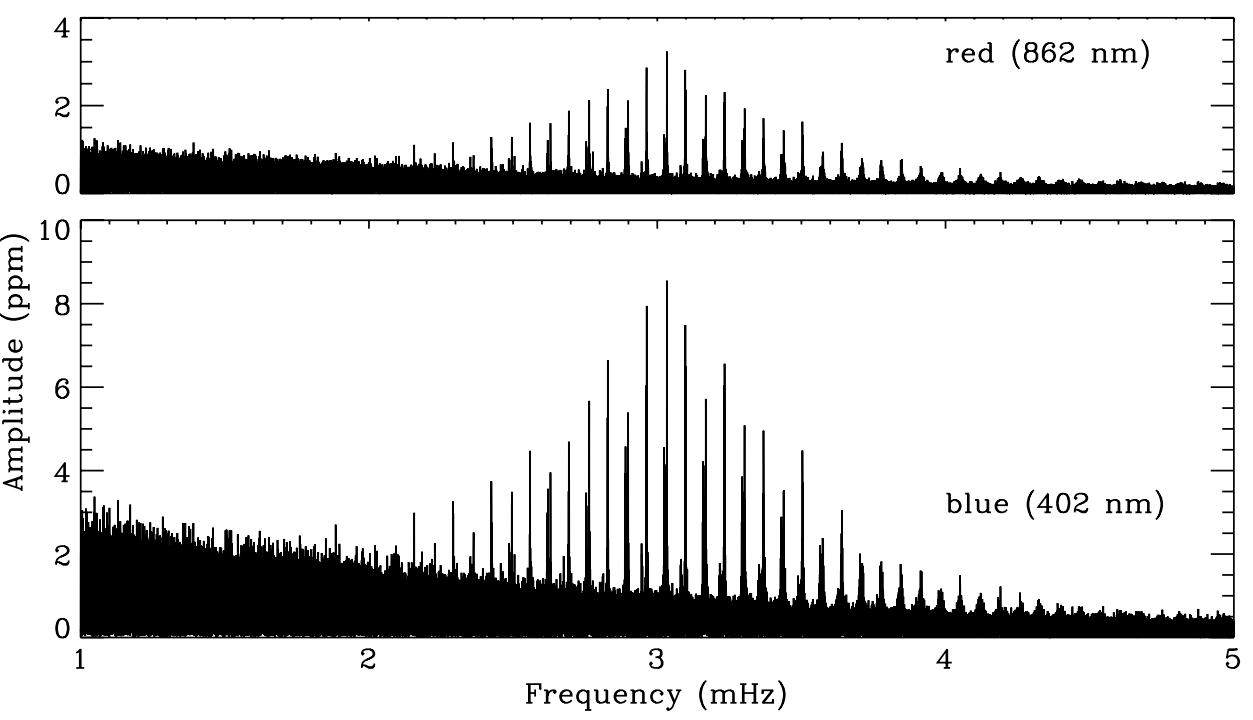

Figure 1 Amplitude spectra of full-disk solar oscillations measured in intensity by the VIRGO instrument on the SOHO spacecraft. The observations are smoothed and rescaled here to show the spectrum corresponding to 30 days. Individual oscillation modes appear as strong peaks rising above a sloping background, which arises from random convective motion on the solar surface. (Adapted from Fröhlich et al. 1997.)

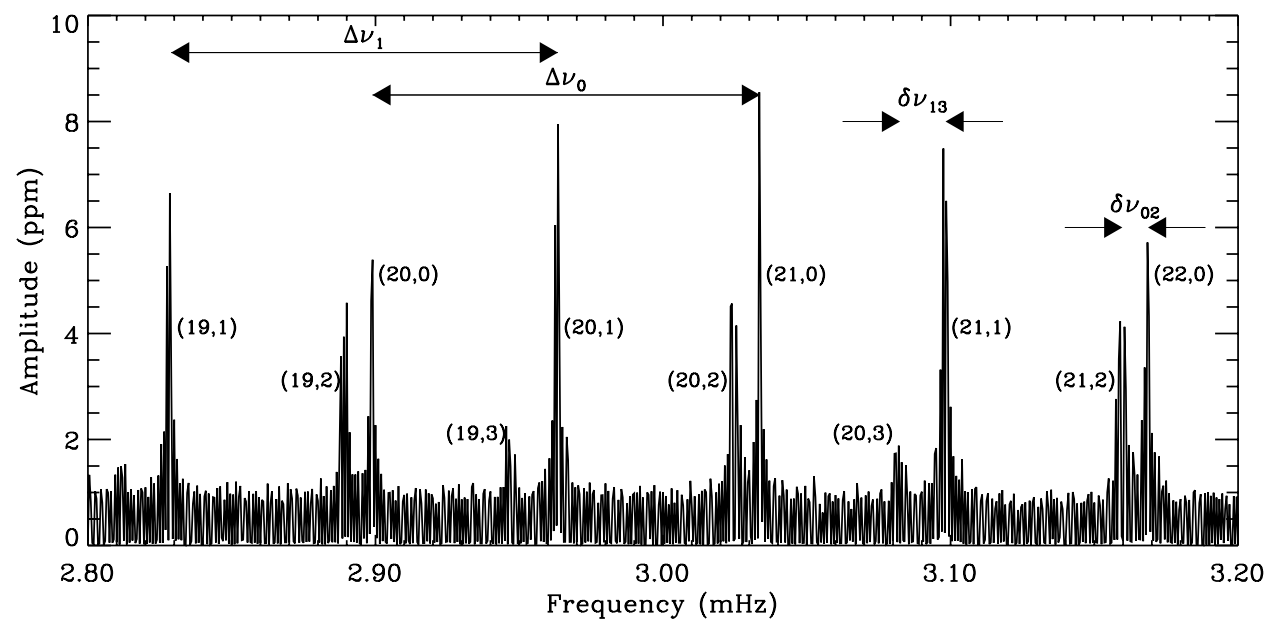

Figure 2 Small section of the solar amplitude spectrum (lower panel of Figure 1), showing $(n, l)$ values for each mode. The large and small separations are indicated. These measure the average density and core composition, respectively, and can therefore be used to infer the mass and age of a star.

together with the similar quantity $\delta v_{13}$. We can further define $\delta v_{01}$ to be the amount by which $l=1$ modes are offset from the midpoint between the $l=0$ modes on either side (not shown in Figure 2). If the asymptotic relation holds exactly, then it follows that $D_{0}=\frac{1}{6} \delta v_{02}=\frac{1}{2} \delta v_{01}=$ $\frac{1}{10} \delta v_{13}$.

In practice, the asymptotic relation does not hold exactly, even for the Sun. For example, the large separation depends on $l$ (hence the separate definitions of $\Delta v_{0}$ and $\Delta v_{1}$ in Figure 2). The large and small separations also depend on frequency. Therefore, a first asteroseismic analysis might involve comparing $\Delta v$ and $\delta v_{02}$ with models (e.g., Brown et al. 1994; Gough 2003), but a full analysis will include a detailed comparison of individual frequencies.
Significant departures from the asymptotic relation are expected for evolved stars. As discussed by Christensen-Dalsgaard et al. (1995), the steep maximum in the local buoyancy frequency at the edge of the inert helium core gives rise to modes behaving like trapped internal gravity waves (so-called g modes; e.g., Dziembowski \& Pamyatnykh 1991; Audard et al. 1995). The core contracts as the star evolves, leading to an increase in the buoyancy frequency and hence in the frequencies of the g modes. Meanwhile, the p-mode frequencies decrease with time as the mean density decreases. When the frequency of a $\mathrm{g}$ mode approaches that of a $\mathrm{p}$ mode, the two modes undergo an avoided crossing (also called mode bumping) where they exchange physical nature. This is illustrated in Figure 3, which shows 


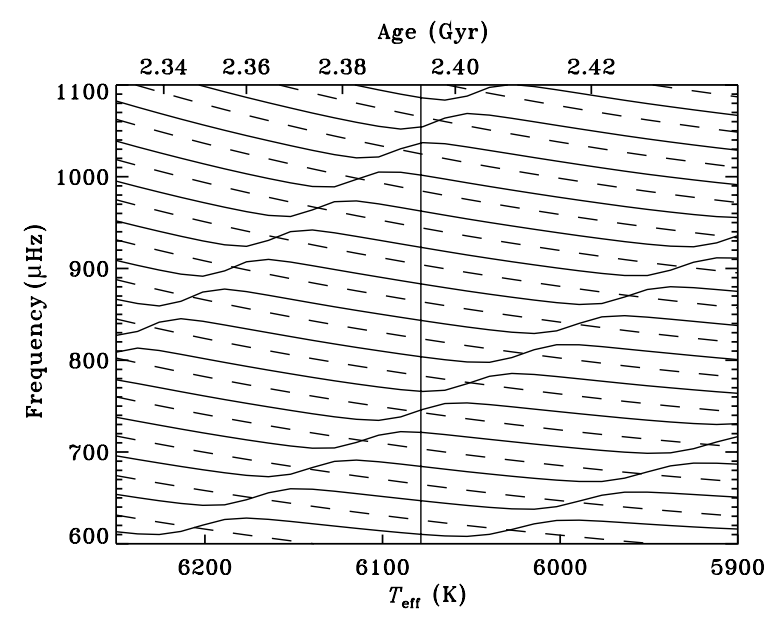

Figure 3 Evolution of adiabatic frequencies with age of a model of mass $1.60 M_{\odot}$, where age is measured by the effective temperature. The dashed lines correspond to modes of degree $l=0$, and the solid lines to $l=1$. The vertical solid line indicates the $T_{\text {eff }}$ of $\eta$ Boo. Figure taken from Christensen-Dalsgaard et al. (1995).

frequency as a function of $T_{\text {eff }}$ for an evolving $1.6 M_{\odot}$ star. Radial modes $(l=0)$, shown by dashed lines, closely follow equation (1); the decrease in frequency with age reflects the increasing stellar radius. The same trend is seen for modes with $l=1$ (solid lines), but here we also see the effect of the additional set of $g$ modes, whose frequencies increase with age. At the avoided crossings the modes take on mixed characters between pure $\mathrm{p}$ and pure $\mathrm{g}$ modes. The frequencies of these mixed modes are extremely sensitive to the evolutionary state and are therefore very interesting, provided they are excited to observable amplitudes. Observational evidence for mixed modes, in the form of frequencies departing from the asymptotic relation, has been seen in subgiants such as $\eta$ Boo and $\beta$ Hyi.

We also note that stellar rotation causes modes with $l \geq 1$ to split into multiplets with different values of $m$, with a separation that directly measures the rotation rate averaged over the region of the star that is sampled by the mode. For the Sun, these types of measurements have given important information about the internal rotation (e.g., Chaplin et al. 2001) and it is hoped that similar results will soon be achieved on other stars (see Gizon $\&$ Solanki 2003). The measurements are particularly difficult because they require long time series, in order to resolve the rotational splittings.

Oscillations in the Sun are long-lived compared to their periods, which allows their frequencies to be measured very precisely. However, the lifetime is finite and this results in the peaks in the power spectrum having a Lorentzian profile with a linewidth that indicates the mode lifetime (Toutain \& Fröhlich 1992). Mode lifetimes have not yet been directly measured for other solar-type stars, and a concern remains that some stars may have mode lifetimes so short that rotational splittings - and perhaps even the small and large separations-will not be observable. In the case of red giants, on the other hand, it appears that mode lifetimes have been measured (see Section 4.11).

\section{Observational Techniques}

Stellar oscillations have been observed by their effect on the stellar atmosphere in three ways (see e.g., Kjeldsen 2003): (i) velocity shifts of spectral lines, (ii) variations in total intensity (photometry), and (iii) variations in equivalent widths of temperature-sensitive lines. In all cases, coverage of the target needs to be as continuous as possible to reduce confusion from aliases in the spectral window. ${ }^{1}$

\subsection{Velocity Measurements}

Most of the new results are based on velocity measurements obtained using high-dispersion spectrographs with stable reference sources. The dramatic improvement in Doppler precision over recent years is a direct result of programs to detect planets around other stars. Asteroseismology has benefited tremendously from these advances. Indeed, in one sense it is easier to measure oscillations because the timescales of interest are much shorter than for exoplanets. On the other hand, the amplitudes of oscillations in solar-type stars are extremely small, which is the reason that positive detections have been so difficult.

\subsection{Intensity Measurements}

Intensity measurements have three tremendous advantages: they can be made with extremely simple instrumentation; they use photons across a wide range of wavelengths; and they can be made simultaneously on many stars (although to some extent, multi-object spectroscopy also offers this possibility). Unfortunately, scintillation from the Earth's atmosphere severely limits the precision of ground-based photometry. The best results to date were obtained by Gilliland et al. (1993) in a multi-site campaign using differential photometry of the open cluster M67, but these fell short of providing definite detections. Atmospheric scintillation on the Antarctic plateau may be small enough to make high-precision photometry feasible (Marks 2002), and there are plans to establish an observatory at Dome C (Schmider et al. 2002). The chance for many weeks - or even months of continuous observations represent an additional bonus.

Space is the ideal place to make intensity measurements, given the absence of atmospheric scintillation and the possibility for long periods of uninterrupted observations. The Fine Guidance Sensors on the Hubble Space Telescope were exploited by Zwintz et al. (1999) to perform high-precision photometry, as was the $52 \mathrm{~mm}$ star camera on the failed WIRE satellite (Buzasi 2002). The space missions designed specifically for asteroseismology are MOST (Matthews et al. 2000, launch date June 2003), COROT (Baglin et al. 1998), and Eddington (Roxburgh \& Favata 2003). It is unfortunate that MONS (Kjeldsen et al. 2000) appears unlikely to proceed, due to lack of funding.

\footnotetext{
${ }^{1}$ The times at which observations are made define the window function and the transform of this in the frequency domain is the spectral window.
} 


\subsection{Equivalent Widths}

This method for detecting oscillations was suggested by Kjeldsen et al. (1995, see also Stello 2002). It involves monitoring changes in spectral lines whose equivalent widths (EWs) are temperature sensitive, most notably the hydrogen Balmer lines. Observations of $\eta$ Boo showed good evidence for oscillations (see Section 4.2), while those of $\alpha$ Cen A only produced a tentative detection (Kjeldsen et al. 1999).

Meanwhile, the usefulness of the Balmer lines for measuring oscillations was questioned by Heasley et al. (1996). They cited the study by Ronan et al. (1991) of the wavelength variation of oscillations in the Sun, which showed that the Balmer lines remain stable while the surrounding continuum fluctuates. Indeed, this is precisely the effect that makes Balmer-line EWs so useful (see also Tycner \& Lester 2002).

\subsection{Comparison of the Methods}

Velocity and EW measurements have an important advantage: they are both more sensitive to modes with $l \geq 2$ than are intensity measurements. In both cases, the reason is that the observations have some spatial resolution of the stellar disk, and so the tendency for high-degree modes to cancel is reduced. For velocity measurements, we measure velocities projected onto the line of sight, which gives more sensitivity to the centre of the disk relative to the limb (Christensen-Dalsgaard \& Gough 1982). For EW measurements, at least for the Balmer lines, the centre-to-limb variation of line strengths gives a similar effect (Bedding et al. 1996). As pointed out by these last authors, the different sensitivities to $l$ of the various methods provides a useful tool for mode identification, and this has been applied to $\delta$ Scuti stars (Viskum et al. 1998; Dall et al. 2002; Dall \& Frandsen 2002).

Intensity and EW measurements both detect temperature changes, and so are much more sensitive to the stellar background that arises from granulation. This is seen in intensity observations of the Sun (Figure 1) as background power rising towards low frequencies, and has been detected in EW observations of $\alpha$ Cen A (Kjeldsen et al. 1999). Although this power contains information about stellar convection that might usefully be compared with models (e.g., Trampedach et al. 1998), for asteroseismology it represents an unwanted and fundamental noise source. Velocity observations are far less sensitive to the stellar background, which is a very important advantage.

Finally, we note one disadvantage of velocity measurements over the other methods: the precision is degraded by stellar rotation, due to line broadening. Of the stars discussed here, only $\eta$ Boo is rotating rapidly enough for this effect to be significant.

At this stage, it seems clear that measuring velocities is the method of choice for ground-based observations. However, the observations require high-resolution spectroscopy with extremely high precision, which is only

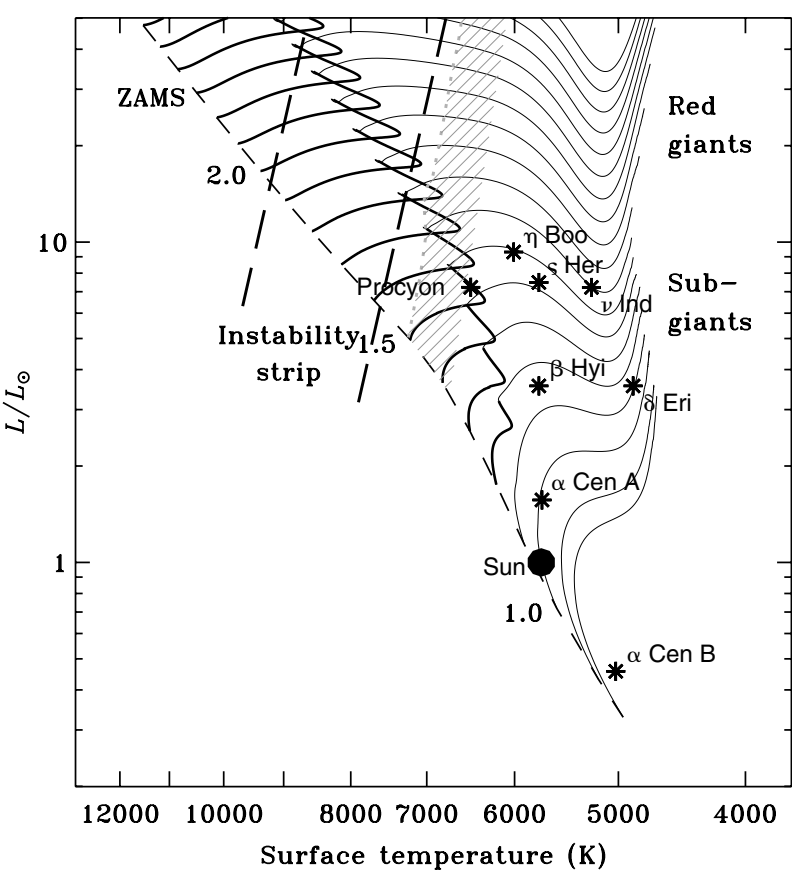

Figure 4 H-R diagram showing some of the stars discussed in the text.

achievable on a handful of instruments. EW measurements may have a place in observing rapid rotators, for which velocity measurements become less precise. Intensity measurements are planned for space missions, and also for observations from Antarctica. Of course, photometry will also continue to be the main method of observing high-amplitude oscillators such as semiregulars (Section 4.11).

\section{Results for Individual Stars}

Early attempts to measure solar-like oscillations, including some claims for detections, have been reviewed by Brown \& Gilliland (1994) and Kjeldsen \& Bedding (1995). More recent reviews appear in conference proceedings and these can be used to follow the development of the field (Frandsen 1997; Kjeldsen \& Bedding 1997; Brown 1998; Bedding \& Kjeldsen 1998; Brown 2000; Kjeldsen \& Bedding 2000; Bouchy \& Carrier 2003). Here we discuss results on some individual stars, with emphasis on the most recent results. The positions in the H-R diagram of most of these stars are shown in Figure 4. Most of the observations discussed here were made in velocity. The exceptions are the EW observations of $\eta$ Boo and the photometric observations of $\alpha$ Cen A and of the red giants ( $\alpha$ Boo, $\alpha$ UMa and the semiregulars).

This review deals mostly with observational aspects, although papers describing theoretical models of individual stars are noted in the relevant sections. There are several reviews in conference proceedings of the theoretical aspects of asteroseismology of solar-like stars (e.g., Christensen-Dalsgaard 1998; Thompson 2000; Roxburgh 2002). In addition, we refer the reader to the 
comprehensive modelling of oscillation frequencies for intermediate-mass stars by Guenther (2002).

\subsection{Procyon ( $\alpha \mathrm{CMi})$}

Procyon is an F5 subgiant and the second brightest star of near-solar type (the brightest is $\alpha \mathrm{Cen}$ ). Evidence for oscillations in Procyon was presented by Brown et al. (1991), in the form of a broad envelope of excess power in the Fourier spectrum. We have previously argued (Kjeldsen \& Bedding 1995) that this power excess could be due to noise, either from instrumental drift or from stellar background. Since then, additional measurements with the FOE and AFOE spectrographs have continued to show the power excess (Brown 2000), although without revealing the characteristic regular series of peaks. Meanwhile, measurements with the ELODIE spectrograph by Martic et al. (1999, see also Barban et al. 1999), gave clear confirmation of the power excess and also provided evidence for a regular spacing of $\Delta v=55 \mu \mathrm{Hz}$. The power excess has also been seen using the EMILIE and CORALIE spectrographs (Bouchy et al. 2002; Carrier et al. 2002) and it now seems clear that it has a stellar origin, although the signature of p-mode oscillations has not been confirmed. It remains to be seen whether the difficulty in obtaining a power spectrum of Procyon that shows a clear p-mode structure is due to insufficient high-quality data (i.e., confusion from aliases in the spectral window) or to intrinsic properties of the star (i.e., short mode lifetimes). Finally, we note that several recent papers present theoretical models for Procyon which are consistent with the above-mentioned value of $\Delta v$ (Chaboyer et al. 1999; Di Mauro \& Christensen-Dalsgaard 2001; Provost et al. 2002).

\section{$4.2 \eta \mathrm{Boo}$}

Kjeldsen et al. (1995) reported evidence for oscillations in the G0 sub-giant $\eta$ Boo, based on measurements of Balmer-line EWs. The observed frequencies, taken with available estimates of the stellar parameters, were in good agreement with theoretical models (ChristensenDalsgaard et al. 1995; Guenther \& Demarque 1996). Particularly interesting was the occurrence in theoretical models - and apparently in the observations — of avoided crossings, in which mode frequencies were shifted from their usual regular spacing by effects of gravity modes in the stellar core (see Section 2). Meanwhile, a search for velocity oscillations in $\eta$ Boo with the AFOE spectrograph by Brown et al. (1997) failed to detect a signal, setting limits at a level below the value expected on the basis of the EW results.

Kjeldsen et al. (2003) presented further observations of $\eta$ Boo, obtained in 1998 in both EW and velocity. They recorded spectra with the Nordic Optical Telescope, which they used to measure equivalent widths of strong temperature-sensitive lines. They also measured velocities using an iodine reference cell at Lick Observatory. Their analysis also included velocity measurements published by Brown et al. (1997) and the original equivalent-width

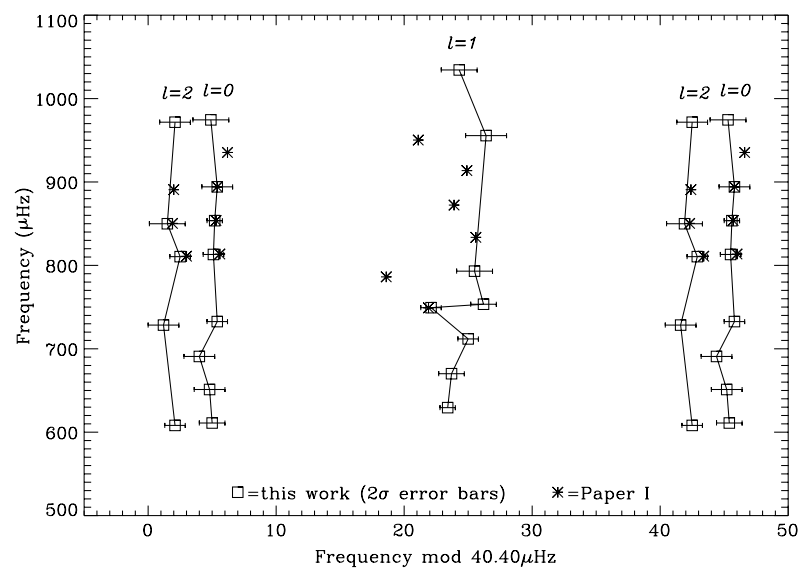

Figure 5 So-called echelle diagram showing the 21 frequencies in $\eta$ Boo, with $2 \sigma$ error bars, together with the 13 frequencies reported by Kjeldsen et al. (1995). Figure from Kjeldsen et al. (2003), and Paper I refers to Kjeldsen et al. (1995).

measurements by Kjeldsen et al. (1995). All four data sets showed power excesses consistent with oscillations, although with a range of amplitudes that presumably reflects the stochastic nature of the excitation. The highest peaks showed regularity with a large separation of $\Delta v=40.4 \mu \mathrm{Hz}$ and they identified 21 oscillation frequencies from the combined data (see Figure 5). The observations indicate that peak oscillation amplitudes in $\eta$ Boo are typically 3-5 times solar. This conclusion is consistent with the upper limits reported by Brown et al. (1997).

Finally, we note that Carrier et al. (2003a, and in preparation) reported velocity measurements using the CORALIE and ELODIE spectrographs that showed a clear excess of power and a frequency spacing of $39.6 \mu \mathrm{Hz}$. We can conclude that oscillations in $\eta$ Boo have been clearly detected and that individual mode frequencies have been identified. The frequencies have been compared with theory by Di Mauro et al. (2003a), who were able to reproduce them using models both with and without convective-core overshooting. Future observations of $\eta$ Boo, particularly with the MOST spacecraft, should measure more oscillation modes with greater frequency precision and permit discrimination between these alternatives.

\section{$4.3 \beta \mathrm{Hyi}$}

A clear power excess in this southern G2 subgiant was detected using velocity measurements with UCLES at the Anglo-Australian Telescope by Bedding et al. (2001) and confirmed with CORALIE by Carrier et al. (2001). Although the amplitudes of the highest peaks were in agreement with theoretical expectations, Gough (2001) has since pointed out that the total observed power was a factor of about 1.9 below expectations. In another development, Bedding et al. (2002) found evidence in the UCLES data for a short-lived, high-amplitude oscillation event. Such events have not been seen in the Sun and, if confirmed as a feature of subgiants, these 'starquakes' would 
make it harder to measure accurate mode frequencies and perform asteroseismology.

The UCLES and CORALIE datasets are now being combined to produce a set of oscillation frequencies (H. Kjeldsen et al., in preparation). Meanwhile, theoretical calculations of frequencies for $\beta$ Hyi have been carried out by Fernandes \& Monteiro (2003) and by Di Mauro et al. (2003b), both of which indicate the occurrence of avoided crossings for modes with $l=1$.

Finally, we note that a long-term trend in radial velocity, possibly indicating a low-mass companion, has been reported by Endl et al. (2002) but not yet confirmed by other observers (e.g., Jones et al. 2002).

\section{$4.4 \zeta \operatorname{Her} A$}

This G0 subgiant was observed in velocity with the ELODIE and CORALIE spectrographs (Morel et al. 2001; Bertaux et al. 2003). Excess power was seen with a possible large separation, but more observations are needed to establish the mode frequencies.

\section{$4.5 \delta$ Eri}

This K0 subgiant was observed in velocity with the CORALIE spectrograph by Carrier et al. (2003b). Again, excess power was seen with a possible large separation, but more observations are needed to establish the mode frequencies.

\section{$4.6 \propto \operatorname{Cen} A$}

As a nearby star with the same spectral type as the Sun, $\alpha$ Cen A is an obvious target for asteroseismology. The clear detection of p-mode oscillations by Bouchy \& Carrier $(2001,2002)$ using velocity measurements with the CORALIE spectrograph was an important breakthrough (see Figure 6). These results confirmed the earlier but less secure detection by Schou \& Buzasi (2000), who

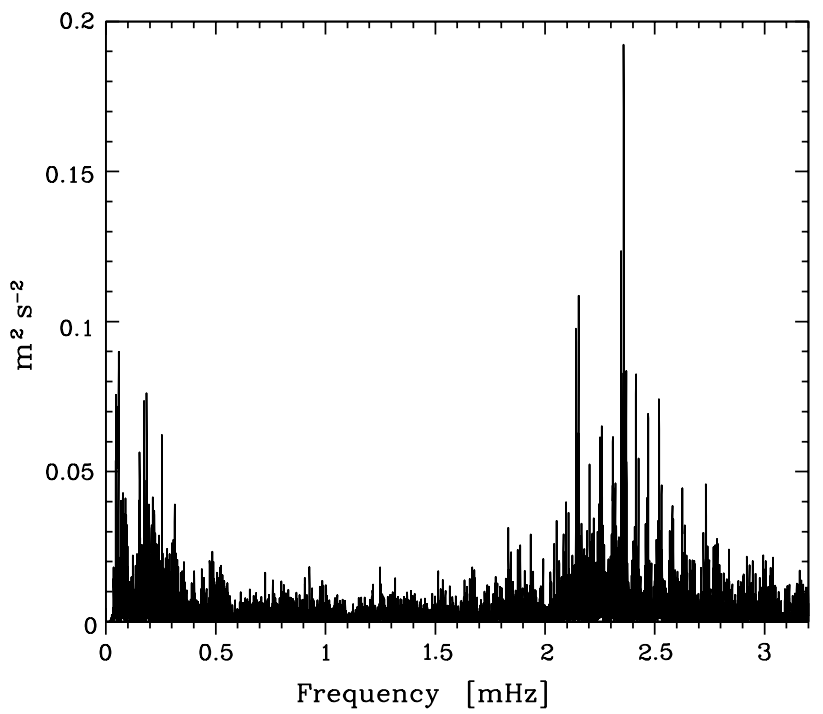

Figure 6 Power spectrum of $\alpha$ Cen A, from Bouchy \& Carrier (2002). used photometry from the WIRE satellite. The CORALIE data have produced frequencies for 28 modes with $l=$ 0-2 and $n=15-25$ (Bouchy \& Carrier 2002) which have been compared with theoretical models by Thévenin et al. (2002) and Thoul et al. (2003). Our own group is analysing additional data on this star taken with the UCLES and UVES spectrographs, and we expect to increase the number of detected frequencies. It is safe to say that seismology of $\alpha$ Cen A will be an important area of research for many years.

\section{$4.7 v$ Ind}

This metal-poor $\mathrm{G}$ subgiant $([\mathrm{Fe} / \mathrm{H}]=-1.4)$ was suggested as a target for asteroseismology by Nissen (1998). Oscillations were detected in velocity using the UCLES and CORALIE spectrographs, and the results are being analysed (T.R. Bedding et al., in preparation; F. Carrier et al., in preparation).

\section{$4.8 \xi$ Hya}

This G7 III giant was observed in velocity with the CORALIE spectrograph (Frandsen et al. 2002; Stello 2002). The observations showed multi-periodic oscillations consistent with radial $(l=0)$ oscillations (see also Teixeira et al. 2003). Using a stochastic excitation model, Houdek \& Gough (2002) found fair agreement between estimated velocity amplitudes and the observations. More work is still to be done, but it seems clear that p-mode oscillations have been detected in this star.

\subsection{Arcturus $(\alpha$ Boo)}

This bright K1 III giant is known to be variable in radial velocity on timescales of a few days (Belmonte et al. 1990; Hatzes \& Cochran 1994; Merline 1995), but without good evidence for a regular p-mode structure in the power spectrum. Observations with the star camera on the WIRE satellite show photometric variability that is consistent with solar-like oscillations (Retter et al. 2003), and the power spectrum appears to have a regular series of peaks with a spacing of $0.83 \mu \mathrm{Hz}$ (Figure 7). However, as

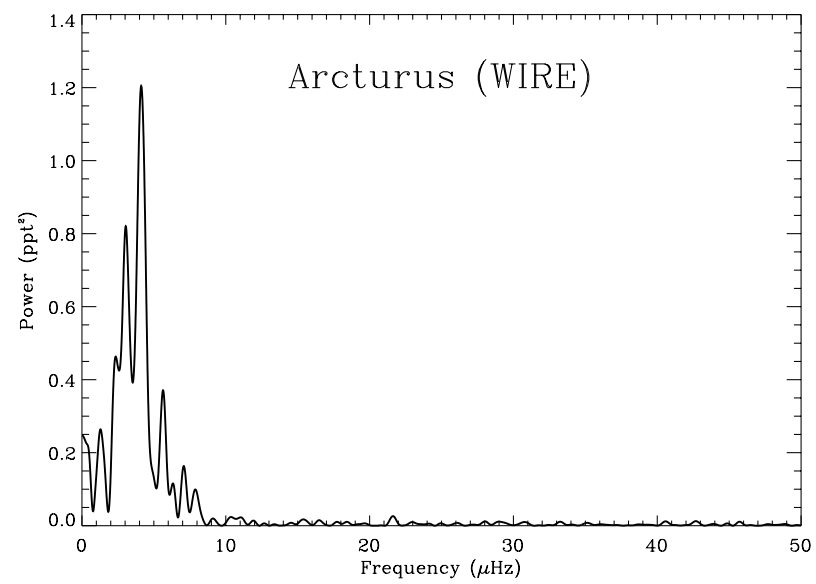

Figure 7 Power spectrum of Arcturus, from WIRE photometry (Retter et al. 2003). 
pointed out by Retter et al. (2003), simulations with pure noise show that the frequencies extracted from a hump of excess power will tend to be regularly spaced, with a separation slightly greater than the formal frequency resolution of the data set (which was $0.64 \mu \mathrm{Hz}$ in this case). Thus, one must be careful in interpreting such a signature, since the envelope of power in Arcturus could also arise from a single mode with a short lifetime. A longer time series is needed to decide this issue.

\section{$4.10 \propto U M a$}

This K0 III giant was observed in photometry with the star camera on the WIRE spacecraft by Buzasi et al. (2000), who interpreted the variability as being due to p-mode oscillations. They reported the apparent detection of ten oscillation modes with a mean separation of $2.94 \mu \mathrm{Hz}$. However, as pointed out in the previous section, care must be taken in interpreting such a signature.

Little work has been done on theoretical models for red giants. In response to the WIRE results, models for $\alpha$ UMa were calculated by two groups, but with inconsistent results. Guenther et al. (2000) were able to match most of the observed frequencies with radial modes, while Dziembowski et al. (2001) were unable to explain the observed oscillation properties.

\subsection{Semiregular Variables}

Oscillating red giants with high luminosity — the long period variables - are conventionally divided into Miras and semiregulars. Mira variables have large amplitudes and are very regular, reflecting the nature of the driving process, which is self-excitation via opacity variations. Semiregulars, on the other hand, have lower amplitudes, less regularity and often show two or three periods. In these stars, it seems plausible that there is a substantial contribution from convection to the excitation and damping. Indeed, Christensen-Dalsgaard et al. (2001) have suggested that the amplitude variability seen in semiregulars is consistent with the pulsations being solar-like, i.e., stochastically excited by convection. The subject of Miralike versus solar-like excitation has also been discussed in the context of K giants by Dziembowski et al. (2001).

Power spectra of the light curves of semiregular variables, based on visual magnitude estimates spanning many decades, show clear evidence for stochastic excitation with mode lifetimes ranging from years to decades (Bedding 2003). An example is shown in Figure 8. We expect more results on semiregulars to be found from mining of databases of visual and CCD photometry.

\section{Conclusions}

Now that solar-like oscillations have been detected in several stars, we are in a position to make some definitive statements. The first and most obvious is that solar-like stars really do oscillate, with properties approximately as expected.

Concerning the oscillation amplitudes, recent theoretical calculations have been made by Houdek et al. (1999), Houdek (2002), Houdek \& Gough (2002), and Samadi et al. (2003a,b). The agreement with observations is fairly good, although it still appears that F-type stars (Procyon and stars in M67) oscillate with lower amplitudes than predicted (Kjeldsen \& Bedding 1995; Samadi \& Houdek

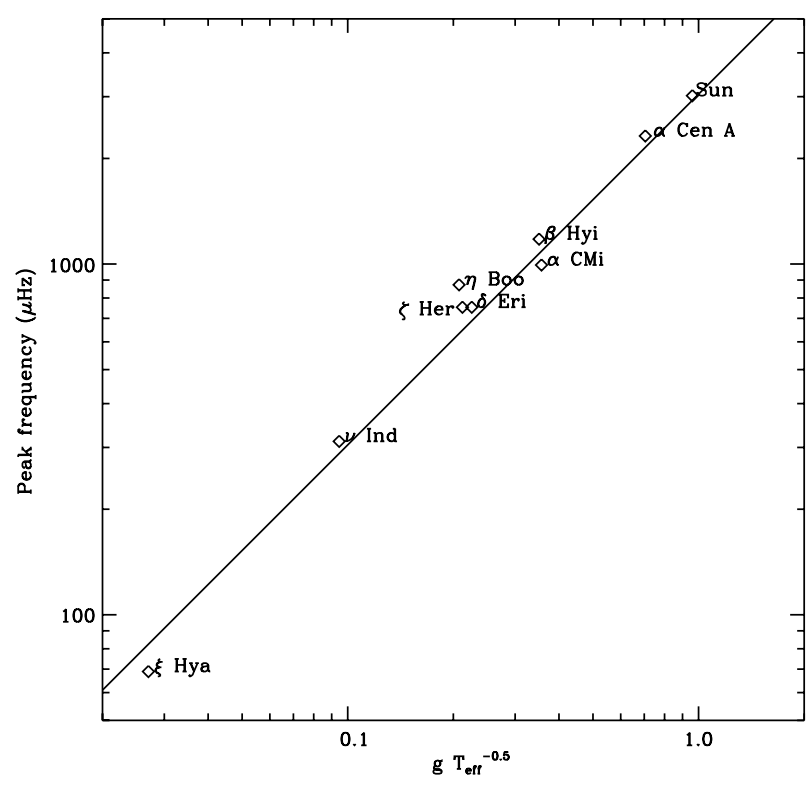

Figure 9 Observed versus expected peak frequencies, where expected values are based on scaling the acoustic cutoff frequency. The diagonal line has a slope of one and passes through the solar value.

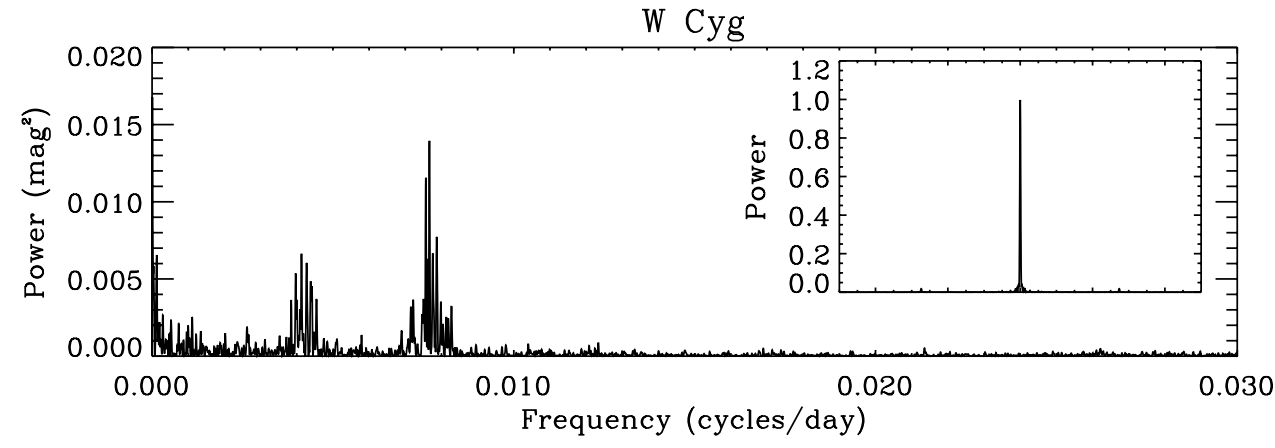

Figure 8 Power spectrum of visual observations of the semiregular variable W Cyg. The inset shows the spectral window. For more examples, see Bedding (2003). 

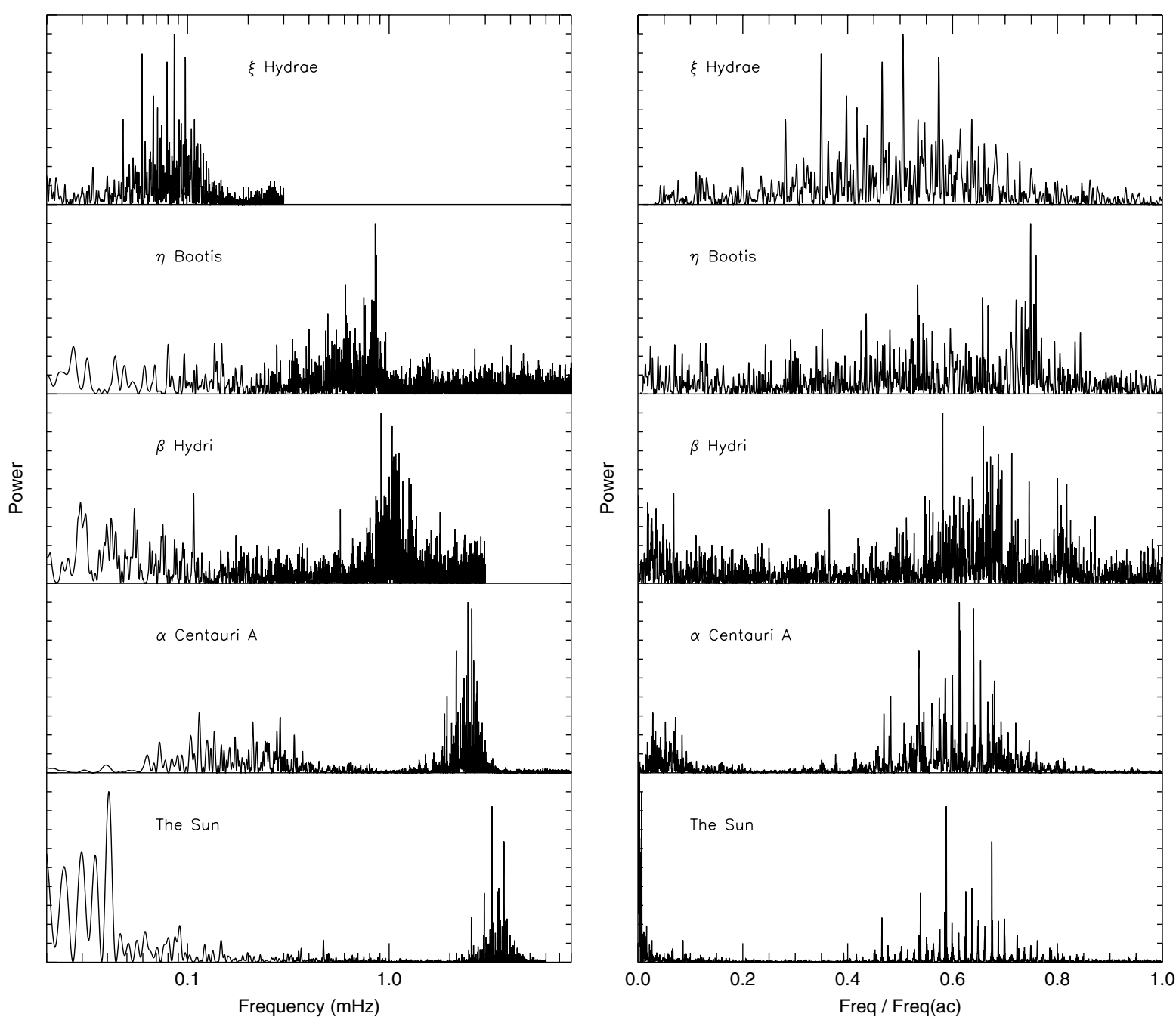

Figure 10 Observed power spectra of oscillations in the Sun and four other stars. On the left, the horizontal axis is observed frequency on a logarithmic scale. On the right, we show frequency (on a linear scale) as a fraction of the acoustic cutoff frequency. The details of the data are as follows: Sun - full-disk velocities from the GOLF instrument on SOHO (Gabriel et al. 1997; note the much lower background compared to the intensity observations in Figure 1); $\alpha$ Cen A — velocities from UVES and UCLES (R. P. Butler et al., in preparation); $\beta$ Hyi — velocities from UCLES and CORALIE (H. Kjeldsen et al., in preparation); $\eta$ Boo - EW from the NOT (Kjeldsen et al. 1995); $\xi$ Hya — velocities from CORALIE (Frandsen et al. 2002). Note that the vertical scales have been normalized to make all stars similar. The actual amplitudes increase up the plot, and the peak power in $\xi$ Hya is about 60 times that of the Sun.

2000). Also, oscillations have yet to be measured in main-sequence stars cooler than the Sun, such as $\alpha$ Cen B.

The observed frequency of maximum power, on the other hand, is in good agreement with theory. The power spectrum of oscillations in the Sun is modulated by a broad envelope whose maximum is at a frequency of $v_{\max } \simeq$ $3 \mathrm{mHz}$. The shape of the envelope and the value of $v_{\max }$ are determined by the excitation and damping. Brown et al. (1991) suggested that $v_{\max }$ in other stars should scale with the acoustic cutoff frequency, $v_{\mathrm{ac}}$. That is, we expect $v_{\max } \propto g T_{\text {eff }}{ }^{-0.5}$ (see also Kjeldsen \& Bedding 1995). As shown in Figure 9, the observed oscillations agree well with this scaling relation. Figure 10 shows the observed oscillation spectra of the Sun and four other stars.

Another result from observations is that avoided crossings in evolved stars seem to occur and offer excellent prospects for detailed analysis. However, they complicate the interpretation of the power spectra and demand observations with few or no gaps, to avoid confusion by aliases in the spectral window.

Further progress from ground-based observations will come from coordinated multi-site campaigns on carefully chosen targets (see Pijpers 2003). We also look forward with great excitement to the dedicated space missions. Many years of effort are now paying off, and we foresee a great future for the study of solar-like oscillations.

\section{Acknowledgments}

This work was supported financially by the Australian Research Council, the Danish Natural Science Research Council, and the Danish National Research Foundation through its establishment of the Theoretical Astrophysics Center. We thank Dennis Stello for helpful comments on the manuscript. 


\section{References}

Aerts, C., Bedding, T. R., \& Christensen-Dalsgaard, J. (eds) 2002, Radial and Nonradial Pulsations as Probes of Stellar Physics, IAU Colloqium 185, ASP Conf. Series 259 (San Francisco: ASP)

Audard, N., Provost, J., \& Christensen-Dalsgaard, J. 1995, A\&A, 297, 427

Baglin, A., et al. 1998, in New Eyes to See Inside the Sun and Stars, Proc. IAU Symposium 185, ed. F.-L. Deubner, J. ChristensenDalsgaard, \& D. W. Kurtz (Dordrecht: Kluwer), 301, http:// www.astrsp-mrs.fr/projets/corot/

Barban, C., Michel, E., Martic, M., Schmitt, J., Lebrun, J.C., Baglin, A., \& Bertaux, J. L. 1999, A\&A, 350, 617

Bedding, T. R. 2003, Ap\&SS, 284, 61

Bedding, T. R., et al. 2001, ApJ, 549, L105

Bedding, T. R., \& Kjeldsen, H. 1998, in Tenth Cambridge Workshop on Cool Stars, Stellar Systems and the Sun, ASP Conf. Series 154, ed. R. A. Donahue, \& J. A. Bookbinder (San Francisco: ASP), 301

Bedding, T. R., et al. 2002, in Radial and Nonradial Pulsations as Probes of Stellar Physics, IAU Colloqium 185, ASP Conf. Series 259, ed. C. Aerts, T. R. Bedding, \& J. Christensen-Dalsgaard (San Francisco: ASP), 464

Bedding, T. R., Kjeldsen, H., Reetz, J., \& Barbuy, B. 1996, MNRAS, 280,1155

Belmonte, J. A., Jones, A. R., Pallé, P. L., \& RocaCortés, T. 1990, ApJ, 358, 595

Bertaux, J.-L., Schmitt, J., Lebrun, J.-C., Bouchy, F., \& Guibert, S. 2003, A\&A, in press

Bouchy, F., \& Carrier, F. 2001, A\&A, 374, L5

Bouchy, F., \& Carrier, F. 2002, A\&A, 390, 205

Bouchy, F., \& Carrier, F. 2003, Ap\&SS, 284, 21

Bouchy, F., Schmitt, J., Bertaux, J.-L., \& Connes, P. 2002, in Radial and Nonradial Pulsations as Probes of Stellar Physics, IAU Colloqium 185, ASP Conf. Series 259, ed. C. Aerts, T. R. Bedding, \& J. Christensen-Dalsgaard (San Francisco: ASP), 472

Brown, T. M. 1998, in Tenth Cambridge Workshop on Cool Stars, Stellar Systems and the Sun, ASP Conf. Series 154, ed. R. A. Donahue, \& J. A. Bookbinder (San Francisco: ASP), 289

Brown, T. M. 2000, in The Third MONS Workshop: Science Preparation and Target Selection, ed. T. Teixeira, \& T. R. Bedding (Aarhus: Aarhus Universitet), 1, available via the ADS

Brown, T. M., Christensen-Dalsgaard, J., Weibel-Mihalas, B., \& Gilliland, R. L. 1994, ApJ, 427, 1013

Brown, T. M., \& Gilliland, R. L. 1994, ARA\&A, 32, 37

Brown, T. M., Gilliland, R. L., Noyes, R. W., \& Ramsey, L. W. 1991, ApJ, 368, 599

Brown, T. M., Kennelly, E. J., Korzennik, S. G., Nisenson, P., Noyes, R. W., \& Horner, S. D. 1997, ApJ, 475, 322

Buzasi, D. L. 2002, in Radial and Nonradial Pulsations as Probes of Stellar Physics, IAU Colloqium 185, ASP Conf. Series 259, ed. C. Aerts, T. R. Bedding, \& J. Christensen-Dalsgaard (San Francisco: ASP), 616

Buzasi, D. L., Catanzarite, J., Laher, R., Conrow, T., Shupe, D., Gautier III, T. N., \& Kreidl, T. 2000, ApJ, 532, L133

Carrier, F., Bouchy, F., \& Eggenberger, P. 2003a, in Asteroseismology Across the HR Diagram, ed. M. J. Thompson, M. S. Cunha, \& M. J. P. F. G. Monteiro (Dorerecht: Kluwer), in press

Carrier, F., et al. 2001, A\&A, 378, 142

Carrier, F., Bouchy, F., Kienzle, F., \& Blecha, A. 2002, in Radial and Nonradial Pulsations as Probes of Stellar Physics, IAU Colloqium 185, ASP Conf. Series 259, ed. C. Aerts, T. R. Bedding, \& J. Christensen-Dalsgaard (San Francisco: ASP), 468

Carrier, F., et al. 2003b, in Asteroseismology Across the HR Diagram, ed. M. J. Thompson, M. S. Cunha, \& M. J. P. F. G. Monteiro (Dorerecht: Kluwer), in press

Chaboyer, B., Demarque, P., \& Guenther, D. B. 1999, ApJ, $525, \mathrm{~L} 41$

Chaplin, W. J., Elsworth, Y., Isaak, G. R., Marchenkov, K. I., Miller, B. A., \& New, R. 2001, MNRAS, 327, 1127
Christensen-Dalsgaard, J. 1998, in The First MONS Workshop: Science with a Small Space Telescope, ed. H. Kjeldsen, \& T. R. Bedding (Aarhus: Aarhus Universitet), 17, available via the ADS

Christensen-Dalsgaard, J. 2002, RvMP, 74, 1073

Christensen-Dalsgaard, J., Bedding, T. R., \& Kjeldsen, H. 1995, ApJ, 443, L29

Christensen-Dalsgaard, J., \& Gough, D. O. 1982, MNRAS, 198, 141

Christensen-Dalsgaard, J., Kjeldsen, H., \& Mattei, J. A. 2001, ApJ, 562, L141

Dall, T. H., \& Frandsen, S. 2002, A\&A, 386, 964

Dall, T. H., et al. 2002, A\&A, 385, 921

Di Mauro, M. P., \& Christensen-Dalsgaard, J. 2001, in Recent Insights into the Physics of the Sun and Heliosphere: Highlights from SOHO and Other Space Missions, IAU Symposium 203, ed. P. Brekke, B. Fleck, \& J. B. Gurman (San Francisco: ASP), 94

Di Mauro, M. P., Christensen-Dalsgaard, J., Kjeldsen, H., Bedding, T. R., \& Paternò, L. 2003a, A\&A, in press

Di Mauro, M. P., Christensen-Dalsgaard, J., \& Paternò, L. 2003b, Ap\&SS, 284, 229

Dziembowski, W. A., Gough, D. O., Houdek, G., \& Sienkiewicz, R. 2001, MNRAS, 328, 601

Dziembowski, W. A., \& Pamyatnykh, A. A. 1991, A\&A, 248, L11

Endl, M., Kürster, M., Els, S. H. A. P., Cochran, W. D., Dennerl, K., \& Döbereiner, S. 2002, A\&A, 392, 671

ESA 2002, Local and Global Helioseismology: The Present and Future, Proc. SOHO 12/GONG+ 2002 Workshop, SP-517

Fernandes, J., \& Monteiro, M. J. P. F. G. 2003, A\&A, 399, 243

Frandsen, S. 1997, in Sounding Solar and Stellar Interiors, Proc. IAU Symposium 181, ed. J. Provost, \& F.-X. Schmider (Dordrecht: Kluwer), 331

Frandsen, S., et al. 2002, A\&A, 394, L5

Fröhlich, C., et al. 1997, SoPh, 170, 1

Gabriel, A. H., et al. 1997, SoPh, 175, 207

Gautschy, A., \& Saio, H. 1995, ARA\&A, 33, 75

Gautschy, A., \& Saio, H. 1996, ARA\&A, 34, 551

Gilliland, R. L., et al. 1993, AJ, 106, 2441

Gizon, L., \& Solanki, S. K. 2003, ApJ, 589, 1009

Gough, D., \& Toomre, J. 1991, ARA\&A, 29, 627

Gough, D. O. 2001, Science, 291, 2325

Gough, D. O. 2003, Ap\&SS, 284, 165

Guenther, D. B. 2002, ApJ, 569, 911

Guenther, D. B., \& Demarque, P. 1996, ApJ, 456, 798

Guenther, D. B., Demarque, P., Buzasi, D., Catanzarite, J., Laher, R., Conrow, T., \& Kreidl, T. 2000, ApJ, 530, L45

Hatzes, A. P., \& Cochran, W. D. 1994, ApJ, 422, 366

Heasley, J. N., Janes, K., Labonte, B., Guenther, D., Mickey, D., \& Demarque, P. 1996, PASP, 108, 385

Houdek, G. 2002, in Radial and Nonradial Pulsations as Probes of Stellar Physics, IAU Colloqium 185, ASP Conf. Series 259, ed. C. Aerts, T. R. Bedding, \& J. Christensen-Dalsgaard (San Francisco: ASP), 447

Houdek, G., Balmforth, N. J., Christensen-Dalsgaard, J., \& Gough, D. O. 1999, A\&A, 351, 582

Houdek, G., \& Gough, D. O. 2002, MNRAS, 336, L65

Jones, H. R. A., Butler, R. P., Marcy, G. W., Tinney, C. G., Penny, A. J., McCarthy, C., \& Carter, B. D. 2002, MNRAS, 337, 1170

Kjeldsen, H. 2003, Ap\&SS, 284, 1

Kjeldsen, H., \& Bedding, T. R. 1995, A\&A, 293, 87

Kjeldsen, H., \& Bedding, T. R. 1997, in Fundamental Stellar Properties: the Interaction between Observation and Theory, Proc. IAU Symposium 189, ed. T. R. Bedding, A. J. Booth, \& J. Davis (Dordrecht: Kluwer), 279

Kjeldsen, H., \& Bedding, T. R. 2000, in Helio- and Asteroseismology at the Dawn of the Millenium, Proc. SOHO 10/GONG 2000 Workshop, ESA SP-464, 361 (astro-ph/0011354)

Kjeldsen, H., et al. 2003, AJ, accepted (astro-ph/0305551) 
Kjeldsen, H., Bedding, T. R., \& Christensen-Dalsgaard, J. 2000, in The Impact of Large-Scale Surveys on Pulsating Star Research, IAU Colloqium 176, ASP Conf. Series 203, ed. L. Szabados, \& D. Kurtz (San Francisco: ASP), 73, see also http://astro.ifa.au.dk/ MONS.

Kjeldsen, H., Bedding, T. R., Frandsen, S., \& Dall, T. H. 1999, MNRAS, 303, 579

Kjeldsen, H., Bedding, T. R., Viskum, M., \& Frandsen, S. 1995, AJ, 109,1313

Marks, R. D. 2002, A\&A, 385, 328

Martic, M., et al. 1999, A\&A, 351, 993

Matthews, J. M., et al. 2000, in The Impact of Large-Scale Surveys on Pulsating Star Research, IAU Colloqium 176, ASP Conf. Series 203, ed. L. Szabados, \& D. Kurtz (San Francisco: ASP), 74, see also http://www.astro.ubc.ca/MOST

Merline, W. J. 1995, PhD Thesis, University of Arizona

Morel, P., Berthomieu, G., Provost, J., \& Thévenin, F. 2001, A\&A, 379,245

Nissen, P. E. 1998, in The First MONS Workshop: Science with a Small Space Telescope, ed. H. Kjeldsen, \& T. R. Bedding (Aarhus: Aarhus Universitet), 99, available via the ADS

Pijpers, F. P. 2003, A\&A, 400, 241

Provost, J., Martić, M., Berthomieu, G., \& Morel, P. 2002, in The First Eddington Workshop on Stellar Structure and Habitable Planet Finding, ESA SP-485, ed. B. Battrick, F. Favata, I. W. Roxburgh, \& D. Galadi, 309

Retter, A., Bedding, T. R., Buzasi, D., Kjeldsen, H., \& Kiss, L. L. 2003, ApJ Letters, submitted

Ronan, R. S., Harvey, J. W., \& Duvall Jr., T. L. 1991, ApJ, 369,549

Roxburgh, I., \& Favata, F. 2003, Ap\&SS, 284, 17

Roxburgh, I. W. 2002, in The First Eddington Workshop on Stellar Structure and Habitable Planet Finding, ESA SP-485, ed. B. Battrick, F. Favata, I. W. Roxburgh, \& D. Galadi, 75

Samadi, R., Goupil, M. J., Lebreton, Y., Nordlund, Å., \& Baudin, F. 2003a, Ap\&SS, 284, 221
Samadi, R., \& Houdek, G. 2000, in The Third MONS Workshop: Science Preparation and Target Selection, ed. T. Teixeira, \& T. R. Bedding (Aarhus: Aarhus Universitet), 27, available via the ADS

Samadi, R., Nordlund, A., Stein, R. F., Goupil, M. J., \& Roxburgh, I. 2003b, A\&A, 403, 303

Schmider, F. X., Fossat, E., Vernin, J., Martin, F., Azouit, M., Agabi, K., Clausse, J. M., \& Grec, G. 2002, in The First Eddington Workshop on Stellar Structure and Habitable Planet Finding, ESA SP-485, ed. B. Battrick, F. Favata, I. W. Roxburgh, \& D. Galadi, 219

Schou, J., \& Buzasi, D. L. 2000, in Helio- and Asteroseismology at the Dawn of the Millenium, Proc. SOHO 10/GONG 2000 Workshop, ESA SP-464

Stello, D. 2002, Master's Thesis, University of Aarhus, Denmark

Teixeira, T., et al. 2003, Ap\&SS, 284, 233

Thévenin, F., Provost, J., Morel, P., Berthomieu, G., Bouchy, F., \& Carrier, F. 2002, A\&A, 392, L9

Thompson, M. J. 2000, in The Third MONS Workshop: Science Preparation and Target Selection, ed. T. Teixeira, \& T. R. Bedding (Aarhus: Aarhus Universitet), 15, available via the ADS

Thompson, M. J., Cunha, M. S., \& Monteiro, M. J. P. F. G. (eds) 2003, Asteroseismology Across the HR Diagram, in press, published in volume 284 of Ap\&SS

Thoul, A., Scuflaire, R., Noels, A., Vatovez, B., Briquet, M., Dupret, M.-A., \& Montalban, J. 2003, A\&A, in press, astro-ph/0303467

Toutain, T., \& Fröhlich, C. 1992, A\&A, 257, 287

Trampedach, R., Christensen-Dalsgaard, J., Nordlund, A., \& Stein, R. F. 1998, in The First MONS Workshop: Science with a Small Space Telescope, ed. H. Kjeldsen, \& T. R. Bedding (Aarhus: Aarhus Universitet), 59, available via the ADS

Tycner, C., \& Lester, J. B. 2002, PASP, 114, 330

Viskum, M., Kjeldsen, H., Bedding, T. R., Dall, T. H., Baldry, I. K., Bruntt, H., \& Frandsen, S. 1998, A\&A, 335, 549

Zwintz, K., Kuschnig, R., Weiss, W. W., Gray, R. O., \& Jenkner, H. 1999, A\&A, 343, 899 